\title{
Strecksehnenverletzungen an Hand und Unterarm
}

\author{
Melanie Fischer, Daniel Gulkin, Joachim Gülke, Martin Mentzel
}

\section{Zusammenfassung}

Der Streckapparat ist ein kompliziertes Geflecht von 2 unterschiedlichen Systemen, nämlich dem intrinsischen und dem extrinsischen, und wird in 9 Zonen eingeteilt. Verletzungen in der Zone 1-3 können i.d.R. konservativ behandelt werden. Verletzungen in den übrigen Zonen bedürfen einer operativen Therapie. Die Dauer der Ruhigstellung richtet sich nach der Lokalisation der Verletzung und nimmt von distal nach proximal ab. Aufgrund enger räumlicher Lagebeziehungen der Fingergelenke zum Skelett sind Begleitverletzungen sorgfältig auszuschließen und ggf. zu behandeln. Posttraumatische Adhäsionen der Sehnen können leicht zu unbefriedigenden Ergebnissen führen.

\section{Injuries of the Extensor Tendons of the Hand and Forearm}

The extensors of the fingers are a complicated network consisting of two different systems - an intrinsic and an extrinsic system - which are subdivided into 9 different zones. Most injuries in zones 1 to 3 can be treated nonoperatively, whereas injuries in zones 4 to 9 usually require surgical treatment. The length of immobilisation varies depending on the location of the injury, with more distal injuries requiring a longer period of immobilisation. Because of the finger joints' proximity to the skeleton, concomitant injuries need to be carefully ruled out or treated, if necessary. Post-traumatic tendon adhesions can potentially cause unsatisfactory results.

\section{Epidemiologie}

Zur Verletzung der Strecksehnen kommt es hauptsächlich durch eine direkte Durchtrennung der Strecksehnen, jedoch können auch Quetschungen, Abrasionsverletzungen, Verbrennungen oder Ausrissverletzungen zur Läsion der Strecksehnen führen. Begleitend zu den Sehnenverletzungen können auch Frakturen vorliegen [1]. Auch geschlossene Verletzungen über den proximalen oder distalen Interphalangealgelenken können Strecksehnenverletzungen nach sich ziehen [2]. Generell sind Strecksehnenläsionen häufiger als Beugesehnenläsionen [3]. Häufig kommt es zum Abriss der Strecksehne bei Arbeitsunfällen, höchste Inzidenzrate findet man unter den 20-29-jährigen Männern [4]. Neben den traumatischen Ursachen darf man

OP-JOURNAL 2015; 31: 152-159

(c) Georg Thieme Verlag KG Stuttgart · New York DOI http://dx.doi.org/10.1055/s-0041-108111 aber auch die degenerativen Rupturen bei rheumatoiden Vorerkrankungen [5] sowie Spontanrupturen nicht vergessen [6].

\section{Anatomie}

Wir unterscheiden beim Streckapparat zwischen einem äußeren und einem inneren System. Die Muskeln des äußeren oder extrinsischen Systems befinden sich am Unterarm, die des inneren oder intrinsischen an der Mittelhand. Die Muskeln des extrinsischen Systems sind die Finger- und Daumenstrecker, die des intrinsischen die Interossei und Lumbricales sowie Muskeln des Daumen- und des Kleinfingerballens. Die Sehnen beider Systeme bilden über Längs- und Querverbindungen ein Geflecht. Die extrinsischen Sehnen verlaufen auf Höhe des Handgelenks in 6 Fächern und stehen am Handrücken über Connexus intertendinei miteinander in Verbindung (siehe Abb. 1). Verletzungen einer Sehne proximal eines solchen Connexus kön- nen deshalb in gewissem Umfang von den Nachbarsehnen kompensiert werden.

Am Finger bilden die extrinsischen Sehnen den Mittelzügel, der an der Basis der Grund- und der Basis der Mittelphalanx sowie über feine Faserzüge an der Basis der Endphalanx inseriert (Abb. 2) [7].

An der Grundphalanx strahlen von beiden Seiten die aponeurotisch auslaufenden Sehnen der intrinsischen Muskeln als Seitenzügel in den Mittelzügel ein und bilden eine sog. Streckhaube, welche die Grundphalanx von 3 Seiten umschließt, und aufgrund der engen Lagebeziehungen bei Verletzungen, aber auch bei Frakturen, kann es leicht durch narbige Verklebungen der Gleitschichten zu Funktionseinschränkungen kommen (siehe Abb. 3) [8].

Die Ausläufer des intrinsischen Systems inserieren an der Basis der Mittel- und der Endphalanx. Im Gegensatz zu den Beugesehnen, die jeweils nur an einer Stelle am Skelett ansetzen, ist der Streckapparat eines Strahles, also über verschiedene Ansatzpunkte, mit jeder der 3 Phalangen eines Fingers verbunden. Daraus resultieren Sehnenabschnitte, die nicht unter direktem Muskelzug stehen. Im Zusammenhang mit der von distal nach proximal zunehmenden Gleitamplitude ermöglicht dies ein konservatives Vorgehen bei gewissen Verletzungen am Endgelenk (Zone 1) und am Mittelgelenk (Zone 3).

Während die Beugesehnen auf alle Gelenke gleichförmig beugend wirken, verhält es sich bei den Strecksehnen komplizierter, indem dieselbe intrinsische Muskel-Sehnen-Einheit auf die Grundgelenke beugend und die Mittel- und Endgelenke streckend wirkt. Aufgrund der geschilderten anatomischen Gegebenheiten führen definierte Verletzungen des Streckapparats zu definierten 


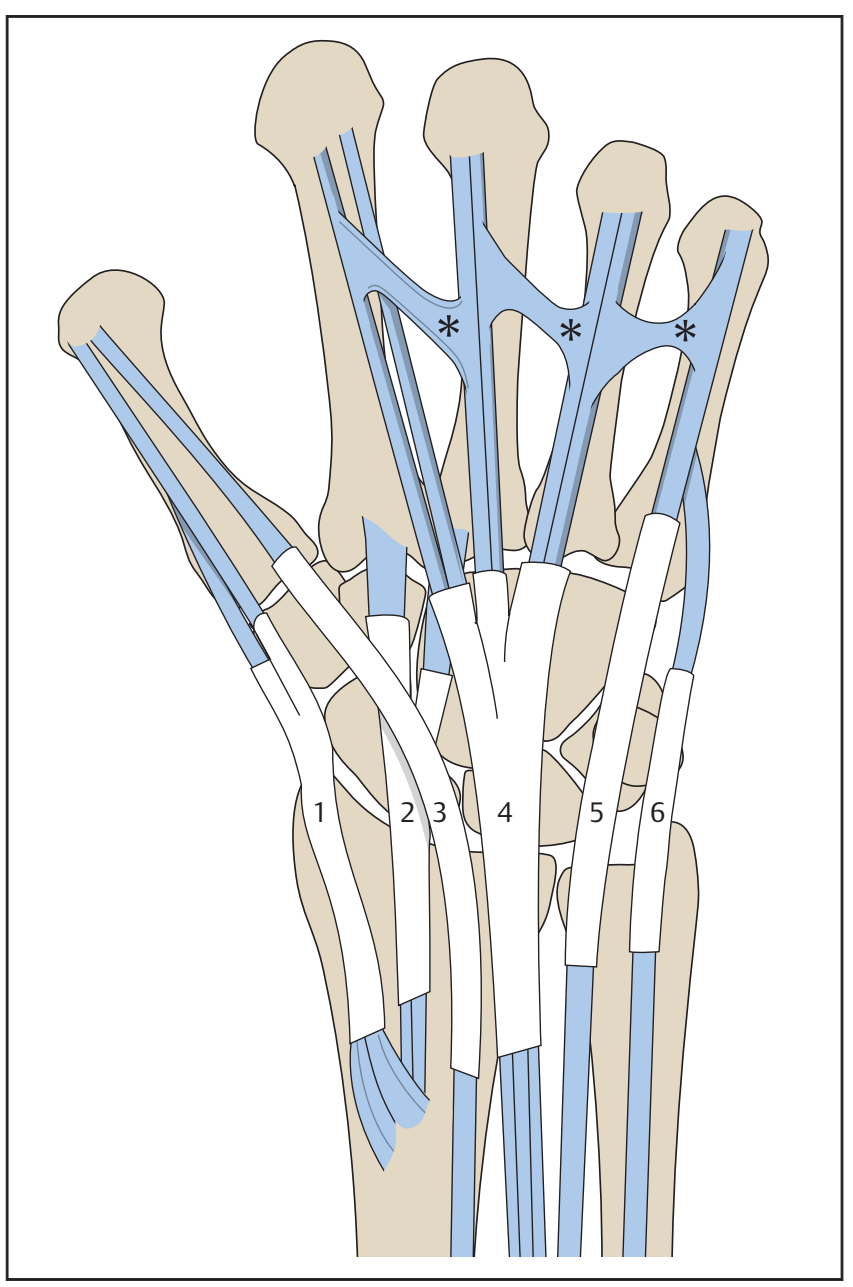

Abb. 1 Anatomie der Strecksehnenfächer. 1 Abductorpollicis-longus-Sehne (APL) und Extensorpollicis-brevis-Sehne (EPB); 2 Extensorcarpi-radialis-longusSehne (ECRL) und Extensor-carpi-radialisbrevis-Sehne (ECRB); 3 Extensor-pollicislongus-Sehne (EPL); 4 Extensor-digitorum-II-IV-Sehnen (ED) und Extensorindicis-Sehne (EI); 5 Extensor-digitiminimi-Sehne (ED V) 6 Extensor-carpiulnaris-Sehne (ECU); * Connexus intertendinei [7].

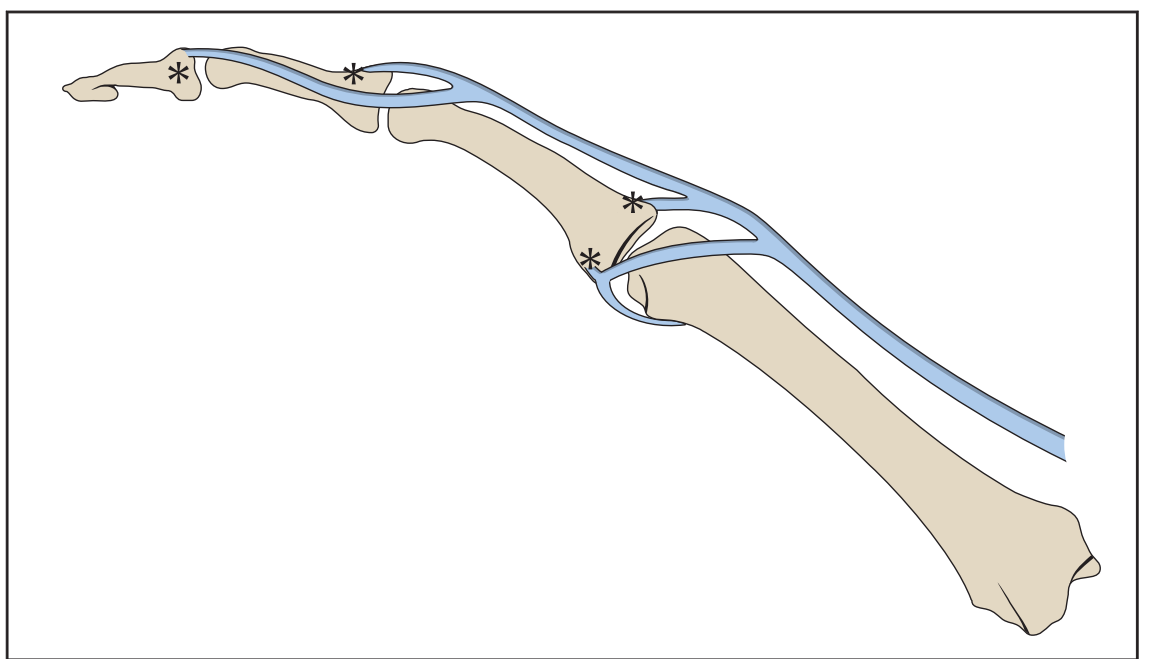

Abb. $2 *$ Schematische Darstellung der Ansatzpunkte des extrinsischen Systems am Skelett.

Deformitäten, d.h. eine Verletzung am Endgelenk und am distalen Mittelglied zum sog. Hammerfinger, eine Verletzung des Mittelzügels über dem Mittelgelenk zur Knopflochdeformation.

\section{Einteilung in Zonen}

Die Einteilung der Strecksehnenverletzungen erfolgt nach Lokalisation der Läsion und wird in 9 Zonen (siehe Abb. 4) unterteilt. Hierbei befinden sich die un- geraden Ziffern über den Gelenken, die geraden dazwischen.

\section{Zone 1 und 2 (distales Interphalangeal-} gelenk und mittlere Phalanx)

Wir unterscheiden einen Abriss der Streckaponeurose bzw. eine reine Sehnendurchtrennung von einem knöchernen Ausriss der Sehne an der Basis der Endphalanx im Sinne einer dorsalen Endphalanxbasisfraktur. Ursächlich sind i.d.R. Distorsionen beim Sport oder bei Stürzen, aber auch Bagatellverletzungen im Haushalt wie das häufig zitierte Verdrehtrauma beim Bettenmachen. Der Finger hängt im Endgelenk meist in einer Beugestellung von $30^{\circ}$ und kann aktiv im Gelenk nicht gestreckt werden (siehe Abb. 5).

Bei der klinischen Untersuchung ist es wichtig, neben der Funktion der Strecksehne auch die Stabilität der Kollateralbänder und die der palmaren Platte zu überprüfen. Radiologisch erfolgt der Frakturausschluss.

Aufgrund der vielfältigen Insertionen des Streckapparats am Skelett kann in der Zone 1 i.d.R. konservativ vorgegangen werden. Das gilt für alle frischen Sehnenrupturen und für knöcherne Ausrisse der Aponeurose ohne wesentliche Dislokation. Die Ruhigstellung erfolgt im Allgemeinen in einer Strecksehnenschiene und dauert meist 8 Wochen (siehe Abb. 6). Anschließend wird die Schiene vorsichtig abtrainiert, aber i.d.R. noch 2-4 Wochen nachts konsequent getragen.

Für knöcherne Sehnenausrisse empfiehlt sich die operative Therapie, wenn das gelenktragende Basisfragment der Endphalanx mehr als die Hälfte der Gelenkfläche beinhaltet und/oder mehr als $2 \mathrm{~mm}$ disloziert ist, und darüber hinaus in allen Fällen, in denen eine Subluxation bzw. Luxation vorliegt. Die Osteosynthese kann mit Schrauben oder Bohrdrähten (siehe Abb. 10), aber auch mit transossären Nähten oder einer Hakenplatte erfolgen. Gegebenenfalls ist auch eine Endgelenksfixation mit Einbringen eines K-Drahtes für zumeist 6 Wochen erforderlich (siehe Abb.7, 8 und 9). Offene Verletzungen werden ebenfalls operativ versorgt. Die Sehnennaht erfolgt mit Uoder Z-Nähten der Stärke $4 \times 0$. Unter Umständen ist eine Endgelenkstransfixation sinnvoll. 


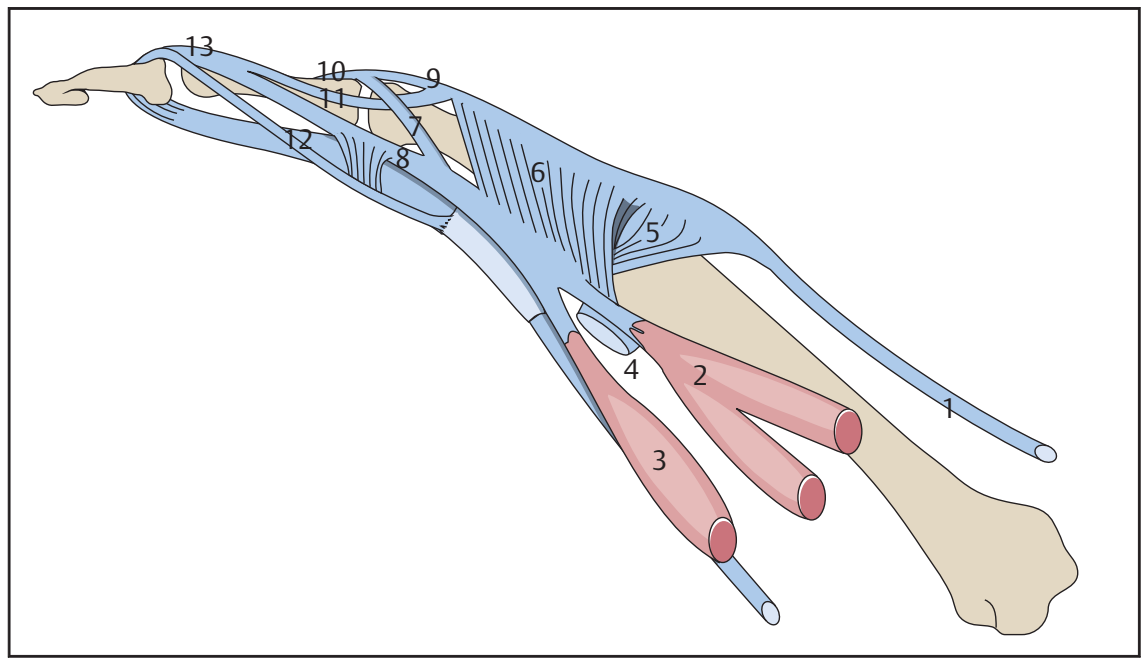

Abb. 3 Schematische Darstellung des Streckapparats der Finger in Seitenansicht von radial. (Nach Buck-Gramcko D. Funktionelle Anatomie. In: Handchirurgie. Band I. Nigst H, Buck-Gramcko D, Millesi H. Hrsg. Stuttgart/New York: Thieme; 1981). 1 Sehne des M. extensor digitorum; $2 \mathrm{M}$. interossei; 3 M. lumbricalis; 4 Lig. metacarpale transversum profundum; 5 Lamina transversa (sagittalis); 6 Lamina intertendinea (Streckhaube); 7 Pars medialis der Interosseussehne (Seitenzügel); 8 Pars lateralis der Interosseussehne (Seitenzügel); 9 Pars medialis der Extensorsehne; 10 Tractus intermedius (Mittelzügel); 11 Pars lateralis der Extensorsehne; 12 Lig. retinaculare obliquum; 13 Endsehne der Streckaponeurose [8].

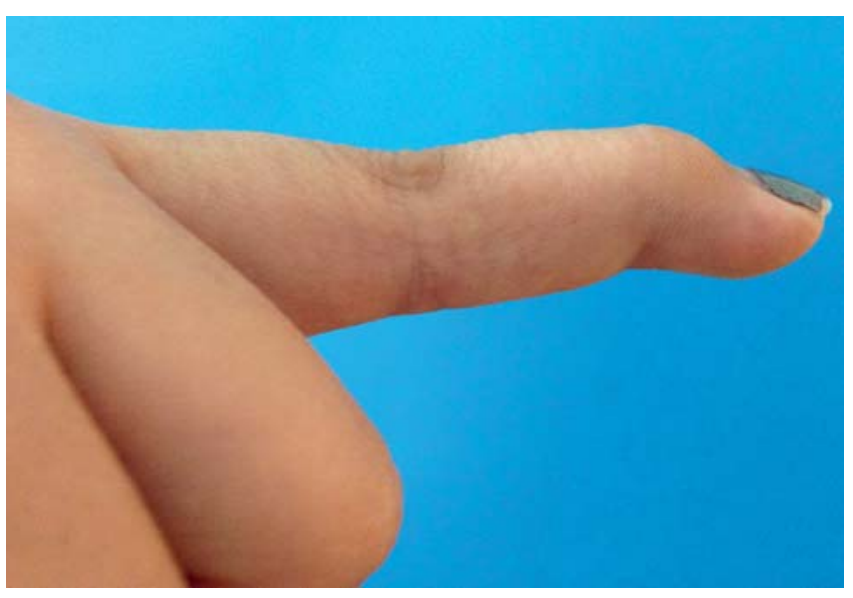

Abb. 5 Gedeckte Strecksehnenruptur Zone 1 mit Streckdefizit im Endgelenk.

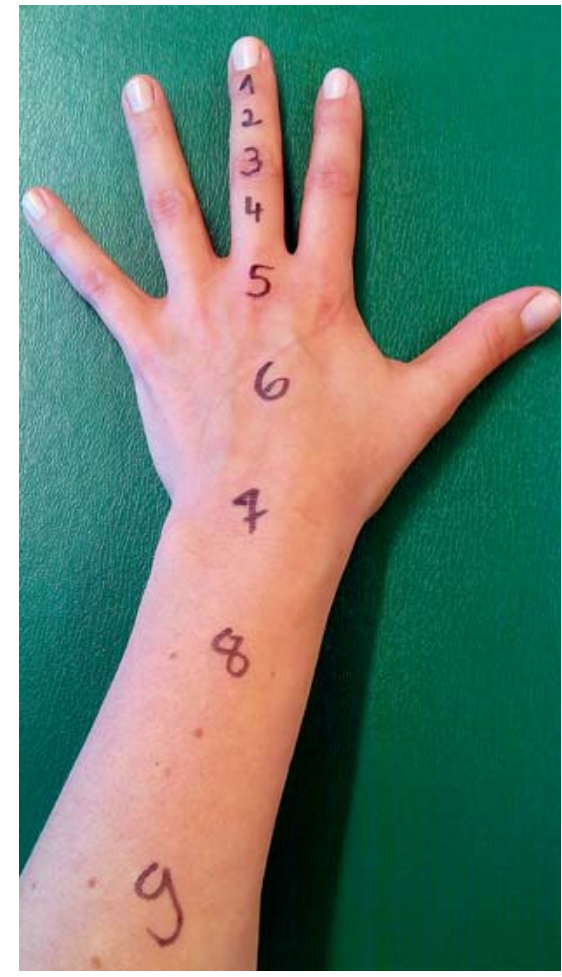

Abb. 4 Zoneneinteilung der Strecksehnen nach Verdan. Man unterscheidet 9 Zonen: Zone 1: Endgelenk; Zone 2: Mittelglied; Zone 3: Mittelgelenk; Zone 4: Grundglied; Zone 5: Grundgelenk; Zone 6: Mittelhand; Zone 7: Handgelenk; Zone 8: muskulotendinöser Übergang und 9: Muskulatur.

\section{Zone 3 (proximales Interphalangeal- gelenk)}

Strecksehnenverletzungen in der Zone 3 betreffen entweder die ganze Streckhaube oder nur Teile, wobei häufig nur der Mittelzügel betroffen ist. Anamnestisch handelt es sich entweder um eine geschlossene Ruptur im Rahmen von Dis-

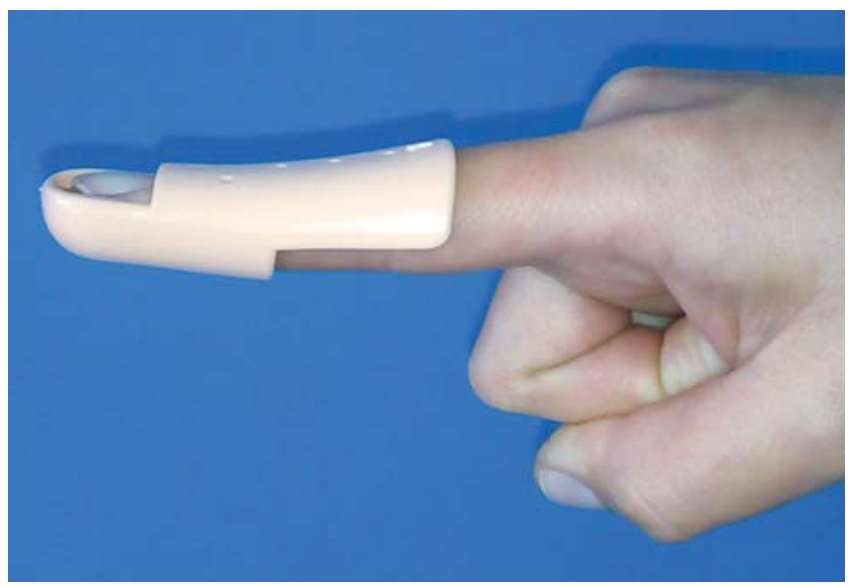

Abb. 6 Stack'sche-Schiene.

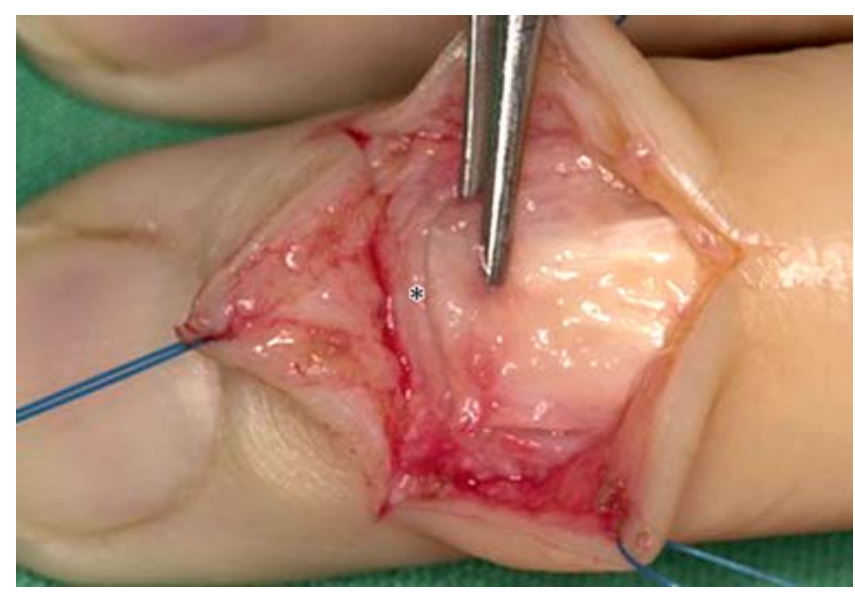

Abb. 7 Veraltete Strecksehnenruptur Zone 1. * Resektion der Sehnennaht. 


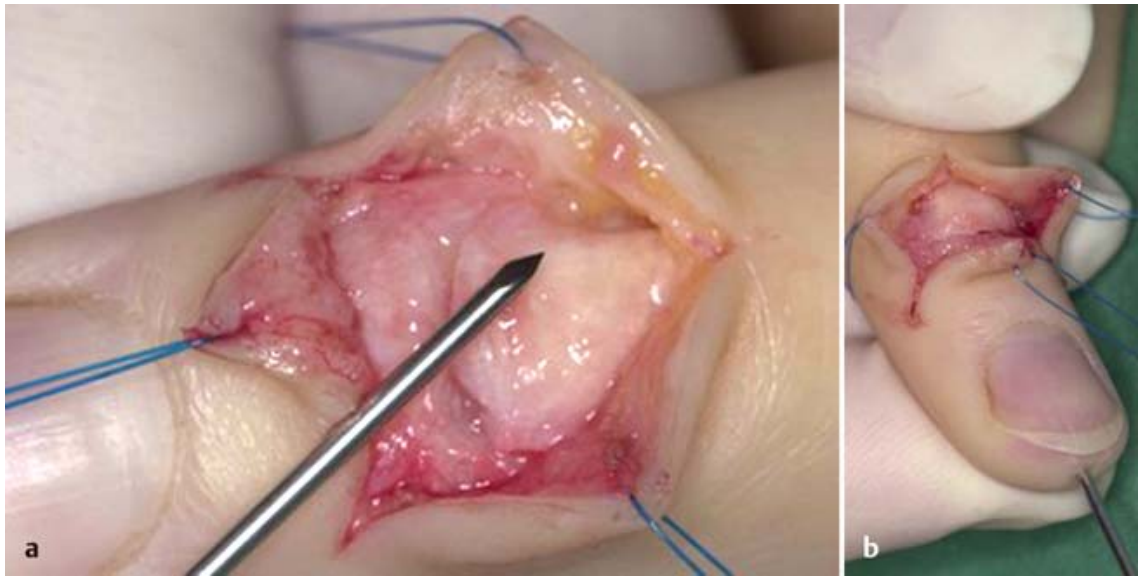

Abb. $\mathbf{8}$ a und $\mathbf{b}$ Temporäre K-Draht-Transfixation.

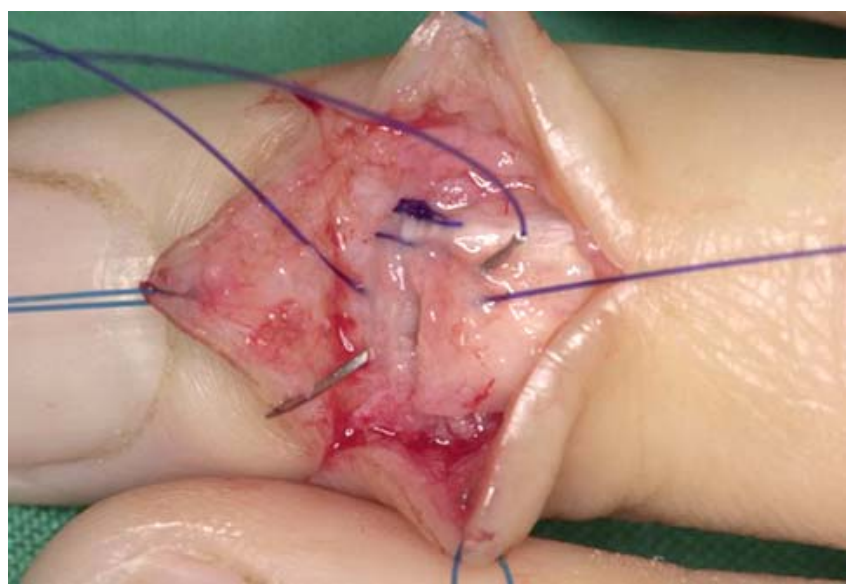

Abb. 9 Z-Nähte zur Adaptation der Strecksehne.
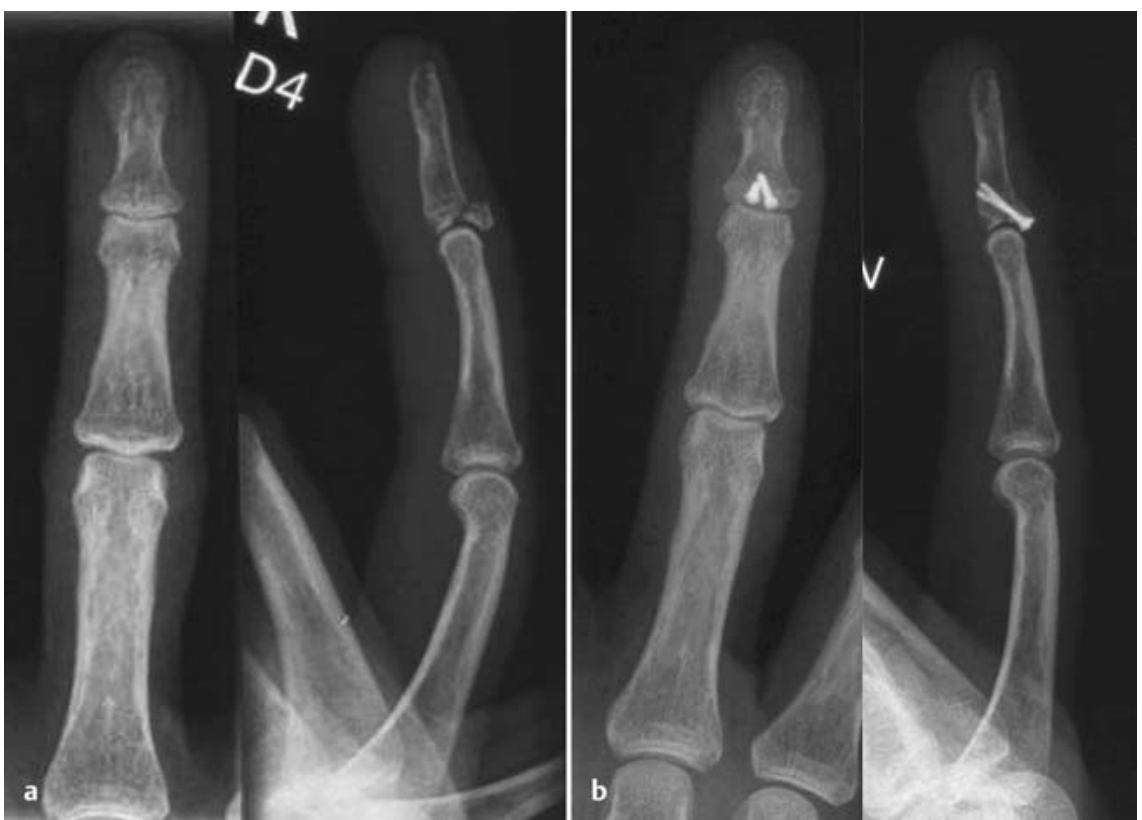

Abb. 10 a und b Knöcherner Strecksehnenausriss mit Schraubenosteosynthesen refixiert. torsionstraumata oder um offene Verletzungen durch scharfe Gegenstände. Klinisch kann der Patient das Mittelgelenk nicht aktiv strecken. Bei einer Teilverletzung kann der Patient das passiv in Streckung gebrachte Mittelgelenk selbstständig gestreckt halten. In diesen Fällen wird konservativ vorgegangen (siehe Abb. 11). Die Dauer der Schienenruhigstellung beträgt 5-6 Wochen.

Kann der Patient das passiv gestreckte Mittelgelenk jedoch nicht selbstständig in Streckung halten, ist von einer weiter gehenden Verletzung der Streckhaube auszugehen, und es besteht die Indikation zur operativen Revision mit Strecksehnennaht und evtl. temporärer Bohrdrahttransfixation des Mittelgelenks (Abb. 12-18).

Gleiches gilt für alle offenen Verletzungen (Abb. 20). Hierbei ist aufgrund der engen räumlichen Lagebeziehungen zwischen der Strecksehne und dem Mittelgelenk immer auch eine Gelenkverletzung in Betracht zu ziehen. Radiologisch wird nach Frakturen und Fremdkörpern gefahndet (siehe Abb. 19).

Intraoperativ wird das eröffnete Gelenk exploriert und gespült. Die Gelenkkapsel kann mit einer monofilen $5 \times 0$-Naht (z.B. PDS) verschlossen werden. Anschließend wird die Strecksehne mit Nähten der Stärke $4 \times 0$ genäht. Zur Anwendung kommen U- oder Z-Nähte und ggf. fortlaufende feinadaptierende Nähte mit Fäden der Stärke $5 \times 0$ oder $6 \times 0$. Ist die Sehne direkt am knöchernen Ansatz durchtrennt, kann sie mit einem Fadenanker refixiert werden (siehe Abb. 1516). Bei Gelenkbeteiligung ist die Gabe eines Antibiotikums empfehlenswert. Die Ruhigstellungszeit beträgt wie bei geschlossenen Verletzungen 5-6 Wochen.

Bei Verletzungen der Zone 3 kann es im Verlauf durch ein Abrutschen der Seitenzügel nach palmar zur sog. Knopflochdeformität kommen.

\section{Zone 4 (Grundglied)}

Bei der Verletzung der Streckhaube in der Zone 4 handelt es sich immer um offene Verletzungen. Da die Streckhaube die Grundphalanx von 3 Seiten umschließt, sind i.d. R. nur Teile von ihr verletzt. Die Durchtrennung eines Seitenzügels wird frühfunktionell behandelt. Mittelzügelverletzungen sowie kombinierte Mittel- und Seitenzügeldurch- 


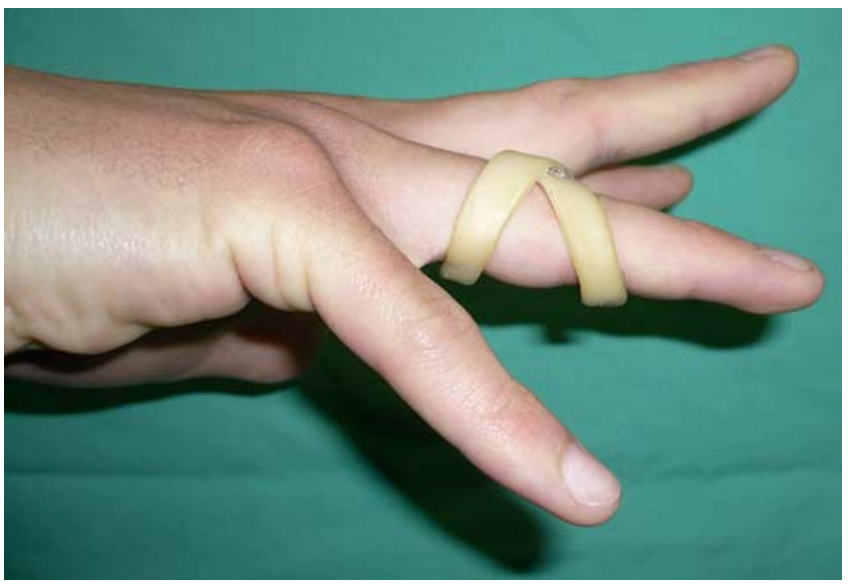

trennungen werden mit U- oder Z-Nähten der Stärke $4 \times 0$ versorgt. Anschließend erfolgte eine Ruhigstellung für 4-5 Wochen.

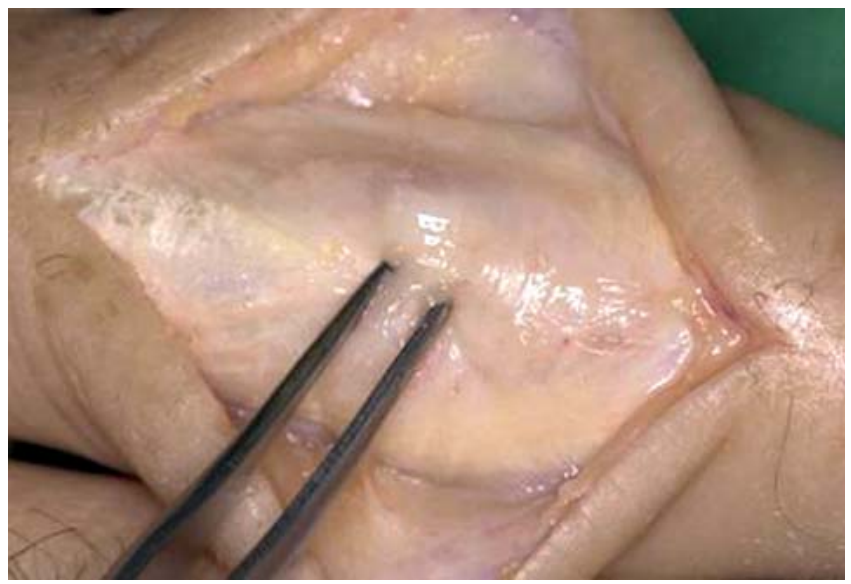

Abb. 13 Blick auf die Streckerhaube des Mittelgelenks.

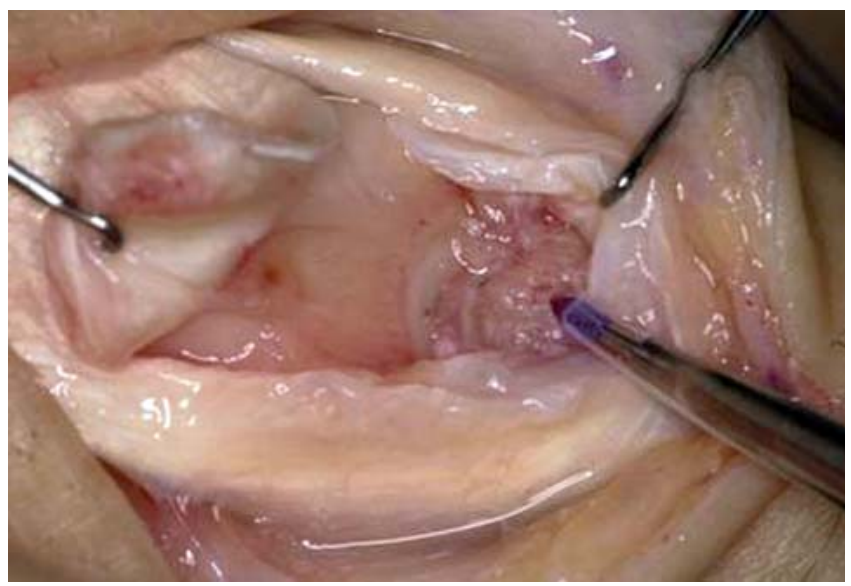

Abb. 15 Einbringen eines Knochenankers am proximalen Mittelglied.
Abb. 11 Stack-Mittelgelenksschiene.

Zone 5 (Metacarpophalangealgelenksebene)

Durchtrennungen der Strecksehnen in der Zone 5 treten im Rahmen offener Verletzungen auf, wobei hier wie in der Zone 3 immer auch Gelenkverletzungen ausgeschlossen werden müssen (Abb. 23-26). Eine typische Konstellation

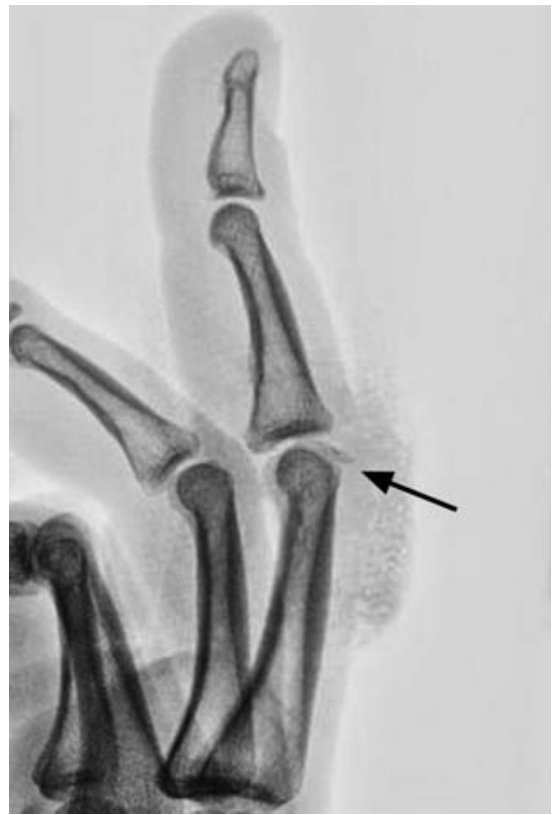

Abb. 12 Knöcherner Ausriss der Streckerhaube über dem Mittelgelenk.
Abb. 14 Blick ins Mittelgelenk mit knöchernem Ausriss des Mittelzügels an der Basis des Mittelglieds.

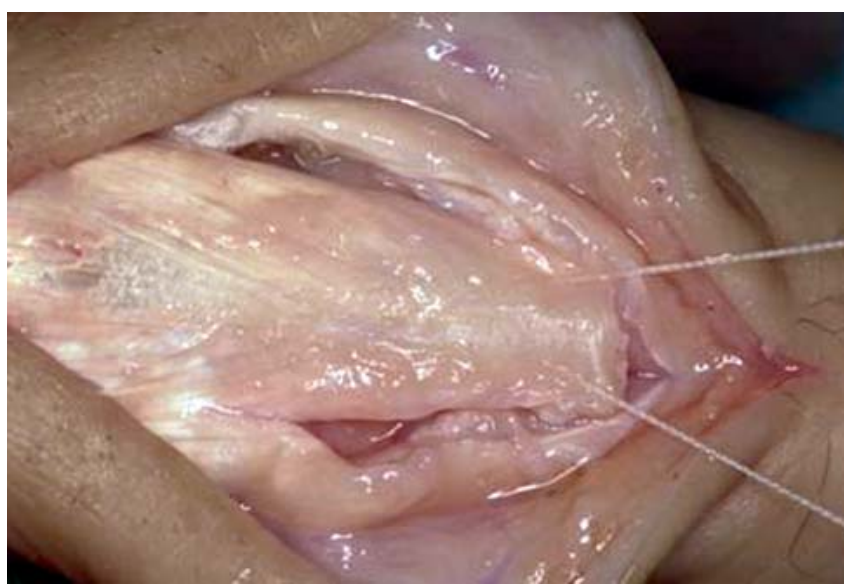

Abb. 16 Refixation des Mittelzügels. 


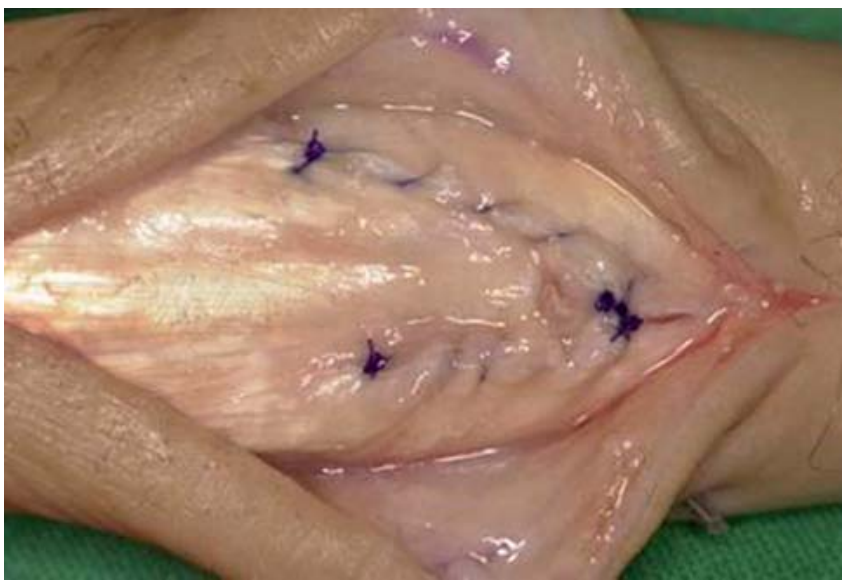

Abb. 17 Fortlaufende Naht.
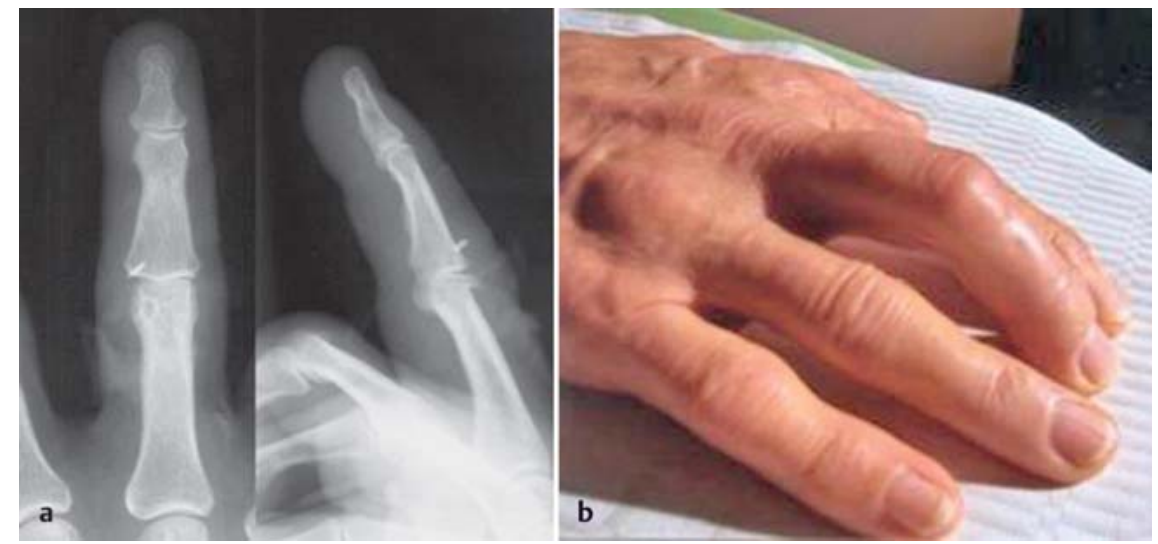

Abb. 19a und b Intraartikuläre Fremdkörperinkorporation (Glassplitter) mit nachfolgendem Infekt und Einsteifung des Mittelgelenks.
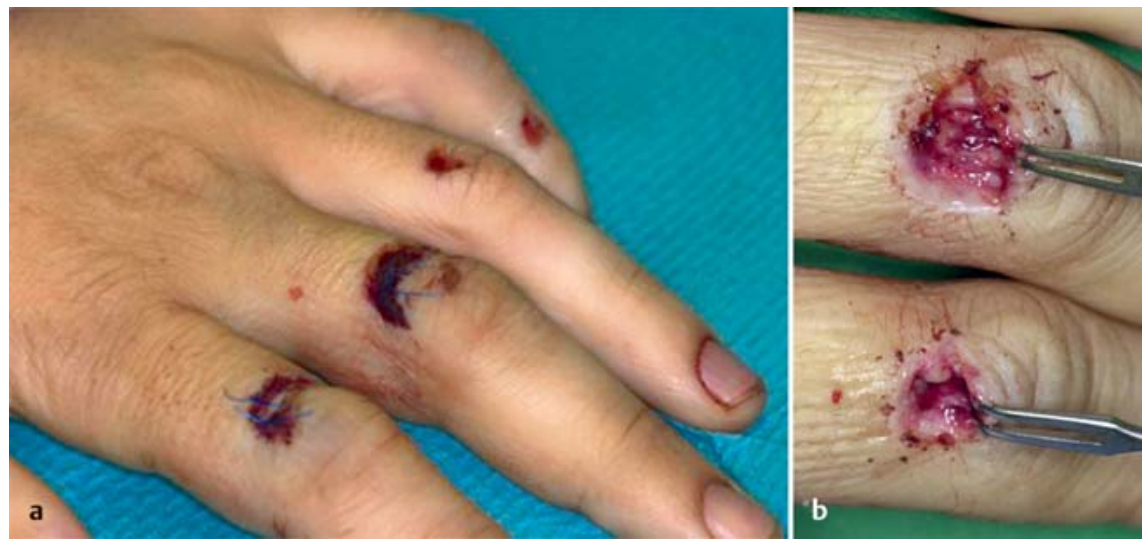

Abb. 20 a und b Riss-Quetsch-Wunden über den Mittelgelenken der Finger DII-IV durch eine Stahltür.

besteht in der Inkorporation abgebrochener Schneidezähne in das Grundgelenk bei Faustschlägen. Das häufige Verkennen dieser Verletzung hat wohl zu der landläufigen Meinung beigetragen, dass Menschenbisse gefährlicher als Hundebisse seien.

Je nach Stärke der Strecksehne, werden U- oder Z-Nähte oder modifizierte Nähte nach Kirchmayr und Kessler mit Kernnaht $(4 \times 0)$ und fortlaufender Feinadaptation $(5 \times 0$ oder $6 \times 0)$ angelegt. Die Dauer der Ruhigstellung beträgt 4 Wochen. Die Schiene wird palmar in sog. Bajonettstellung angelegt, d. h. Unterarm und Finger sind in einer Handgelenkstreckung von $30^{\circ}$ und in einer Grundgelenkbeugung von $30^{\circ}$ parallel ausgerichtet.

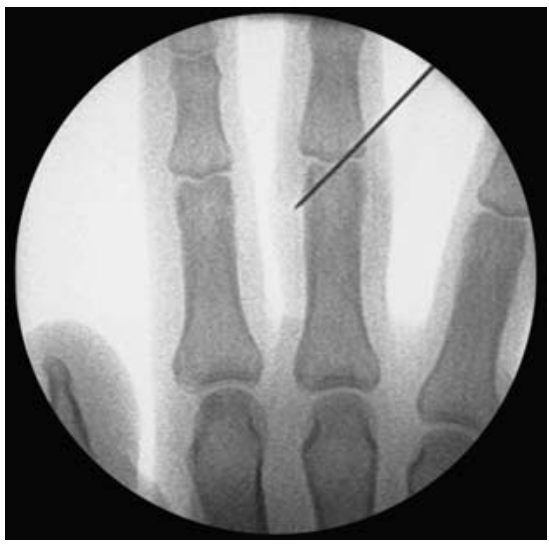

Abb. 18 Temporäre K-Draht-Transfixation.

Darüber hinaus werden in der Zone 5 geschlossene Rupturen der Streckhaube beobachtet, wobei es sich i.d.R. um Rupturen des radialen Seitenzügels mit Luxation des Mittelzügels nach ulnar handelt. Die Behandlung ist operativ durch Naht des Seitenzügels (Nähte der Stärke $5 \times 0$ ). Die Dauer der Ruhigstellung beträgt 3 Wochen.

Typische Lokalisation von Verletzungen durch Faustschläge gegen die Schneidezähne des Gegners.

\section{Zone 6 und 7 (Mittelhand- bis Handgelenk)}

In dieser Zone haben wir es wiederum mit offenen und geschlossenen Verletzungen zu tun. Die Behandlung ist operativ.

Frische traumatische Durchtrennungen der Strecksehnen werden aufgrund des ovalen Querschnitts i.d.R. in der gleichen Technik wie Beugesehnen genäht. Bewährt hat sich die modifizierte Naht nach Kirchmayr und Kessler mit einer Kernnaht $(4 \times 0)$ und fortlaufender Adaptationsnaht $(5 \times 0$ oder $6 \times 0$ ). Die Dauer der Ruhigstellung beträgt 3-4 Wochen. Eine dynamische Behandlung ist möglich. In der Zone 7 ist bei der Rekonstruktion des Retinaculum externum darauf zu achten, dass das Gleiten der genähten Sehnen nicht behindert wird. Evtl. ist eine Erweiterungsplastik sinnvoll.

Geschlossene Rupturen der Strecksehnen in der Zone 6 und 7 werden häufig bei Rheumatikern als Folge von Tendosynovitiden beobachtet. Direkte Sehnennähte sind i.d.R. nicht möglich. Ob die Rekonstruktion durch Brückentransplantate, Umlagerungen oder die Koppelung eines direkten Sehnenstumpfs an eine intakte Nachbarsehne zur Anwen- 


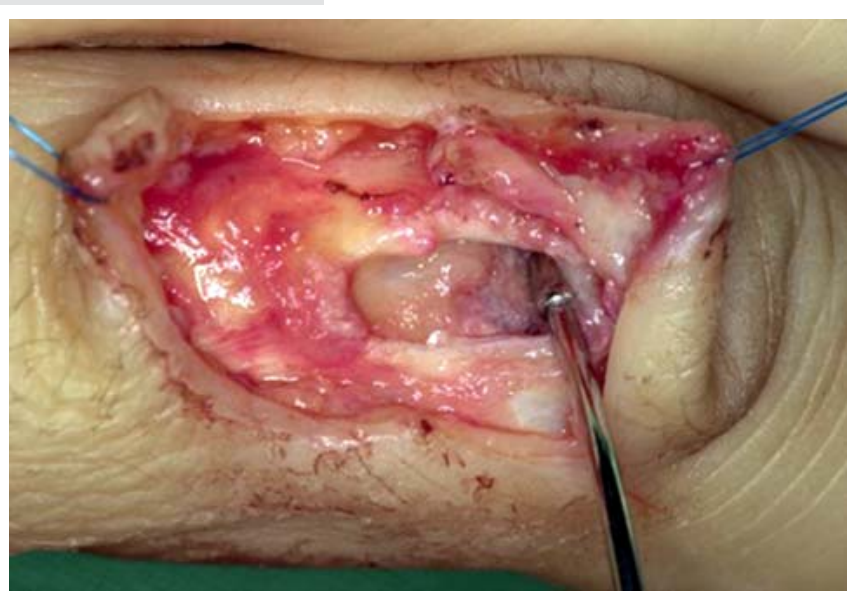

dung kommt, muss im Einzelfall entschieden werden.

Eine typische Entität in der Zone 7 stellt die Ruptur der langen Daumensehne dar. Sie tritt typischerweise bei nicht oder wenig dislozierten, konservativ behandelten distalen Radiusfrakturen aufgrund der Umbauvorgänge nach Einblutung in das 3. Strecksehnenfach auf.
Abb. 21 Mittelzügelverletzung mit Eröffnung des Mittelgelenks.

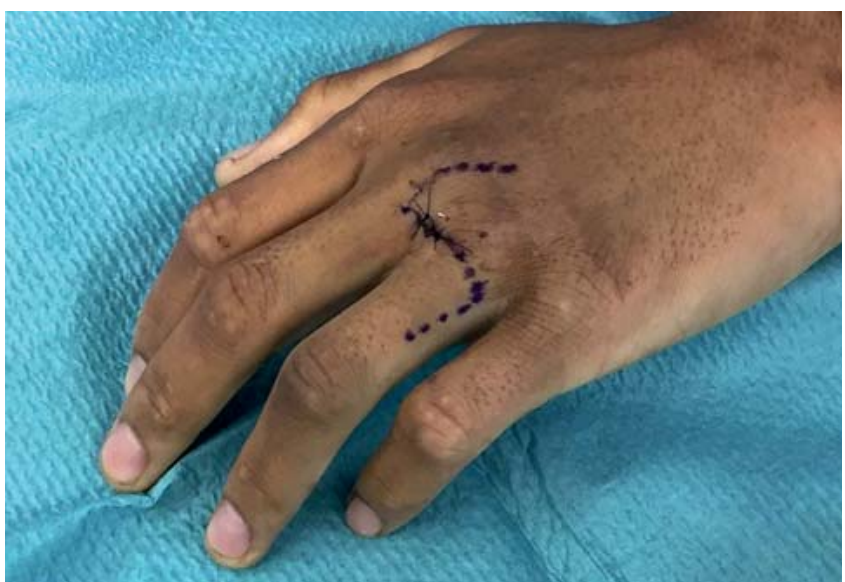

Abb. 23 Schnittführung bei Verletzung über dem Metacarpalgelenk.

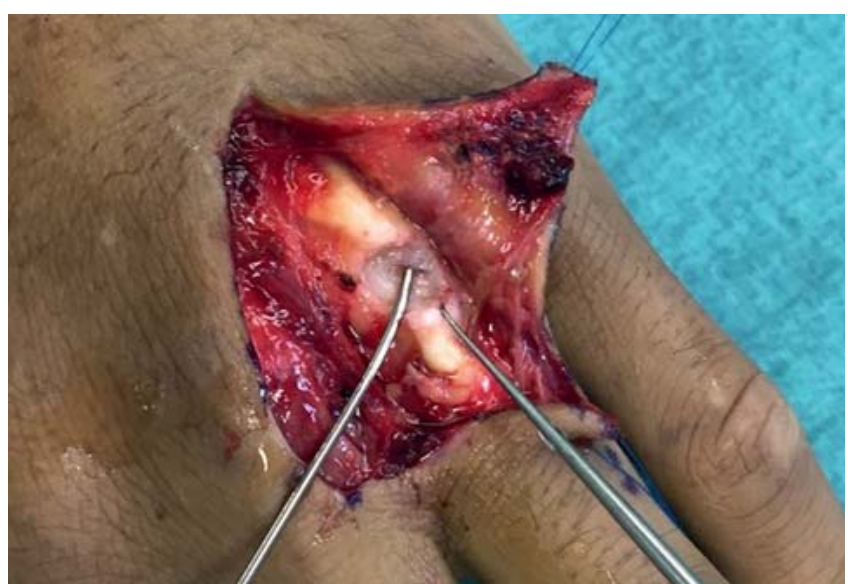

Abb. 25 Läsion mit Eröffnung des Metacarpalgelenks.
Darüber hinaus kann es auch implantatassoziiert iatrogen zu Rupturen der EPL-Sehnen kommen. Eine direkte Sehnennaht ist nicht möglich. Die Rekonstruktion erfolgt nach Umlagerung der Extensor-indicis-proprius-Sehne (Indicistransfer) in Durchflechtungsnahtund Pulvertaft-Technik, wobei intraoperativ auf eine geeignete Vorspannung $\mathrm{zu}$ achten ist. Bei gebeugtem Handgelenk

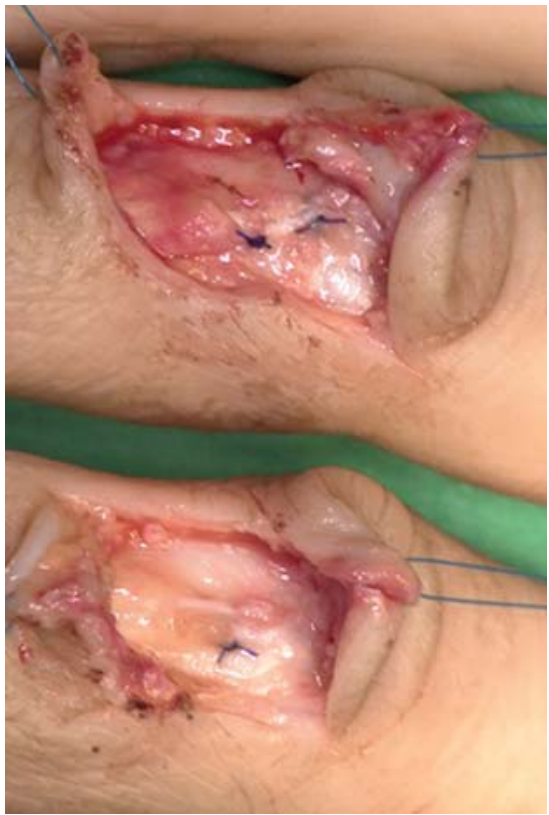

Abb. 22 Adaptierende Z-Nähte.

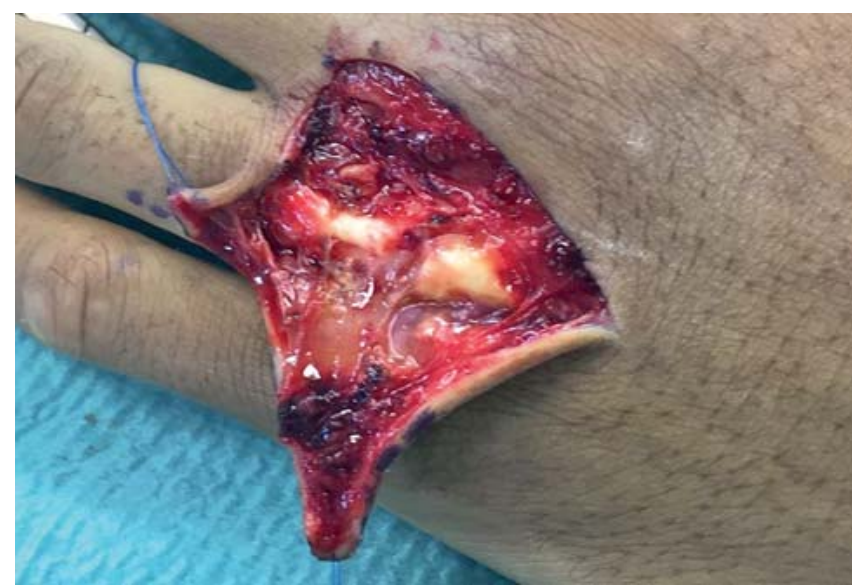

Abb. 24 Durchtrennung der Strecksehne.

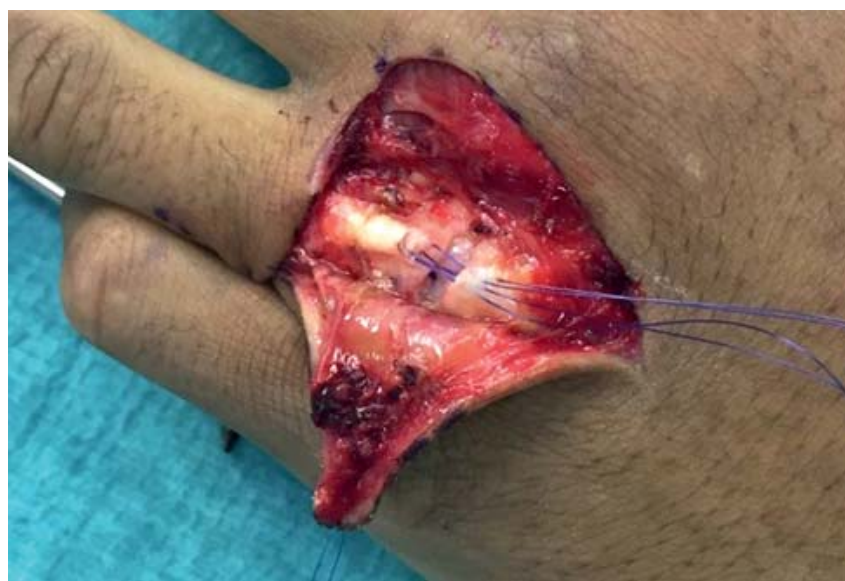

Abb. 26 Anlage von U-Nähten zur Adaptation der Strecksehne. 
sollte das Daumenendgelenk gestreckt sein, bei gestrecktem Handgelenk gebeugt. Die Dauer der Ruhigstellung beträgt 3 Wochen.

Zone 8 (muskolotendinöser Übergang am Unterarm) und Zone 9 (muskulärer Anteil am Unterarm)

Verletzungen der Strecksehnen am muskolotendinösen Übergang oder Verletzungen der Muskulatur treten im Rahmen offener Verletzungen auf. Immer wieder sind erhebliche Gewebszerstörungen anzutreffen, sodass die Versorgung mit Nähten tendenziell schwierig ist. Im muskolotendinösen Übergang kommen U- oder Z-Nähte zur Anwendung, weiter proximal sind i.d.R. nur Fasziennähte möglich. Die Dauer der Ruhigstellung beträgt 2-3 Wochen. Narbige Verklebungen und Nahtinsuffizienzen führen häufig zu einem unbefriedigenden Ergebnis, sodass im Einzelfall im Verlauf durchaus an motorische Ersatzoperationen gedacht werden muss.

\section{Literatur}

${ }^{1}$ Krickhahn M, Kneser U, Kopp J et al. Luxationen und Frakturen der Mittelhand. CHAZ 2004; 5: 1-4

2 Windolf J. Strecksehnenverletzungen der Hand. Unfallchirurg 2006; 109: 659-669

3 Werber KD. Verletzungen des distalen Interphalangealgelenkes. Unfallchirurg 2014; 117 : 327-333

${ }^{4}$ De Jong JP, Nguyen JT, Sonnema AJ et al. The incidence of acute traumatic tendon injuries in the hand and wrist: a 10-year populationbased study. Clin Orthop Surg 2014; 6: 196202

${ }^{5}$ Sakuma Y, Ochi K, Iwamoto T et al. Number of ruptured tendons and surgical delay as prognostic factors for the surgical repair of extensor tendon ruptures in the rheumatoid wrist. J Rheumatol 2014; 41: 265-269

6 Jain A, Goyal N, Mishra P. Spontaneous rupture of EPL and ECRB tendons in a washerwoman: an unusual phenomenon. Hand Surg 2014; 19: 241-244

7 Hoffman R. Checkliste Handchirurgie. Stuttgart, New York: Thieme; 1999

8 Buck-Gramcko D, Hoffmann R, Neumann $R$. Der handchirurgische Notfall. Stuttgart: Hippokrates; 1993

\section{Dr. med. Melanie Fischer}

Fachärztin für Orthopädie und

Unfallchirurgie

Dr. med. Daniel Gulkin

Facharzt für Orthopädie und Unfallchirurgie

PD Dr. med. Joachim Güllke

Facharzt für Orthopädie und Unfallchirurgie, Handchirurgie Prof. Dr. med. Martin Mentzel Sektionsleiter Hand-, Plastische und Mikrochirurgie

Zentrum für Chirurgie

Klinik für Unfall-, Hand-, Plastische und Wiederherstellungschirurgie Universität Ulm

Albert-Einstein-Allee 23

89081 Ulm

Melanie.Fischer@uniklinik-ulm.de 